\title{
Analysis of the genetic relationships from different genetic systems between the amylose content and the appearance quality of indica rice across environments
}

\author{
Guo-Ke Ge, Chun-Hai Shi, Jian-Guo Wu and Zi-Hong Ye \\ Department of Agronomy, Zhejiang University, Hangzhou, China.
}

\begin{abstract}
The genetic relationships between amylose content (AC) and appearance quality traits of indica rice (Oryza sativa L.) were investigated using conditional analysis and unconditional analysis in present experiment. The results from the unconditional analysis indicated that $\mathrm{AC}$ of rice positively correlated with brown rice (BR, i.e., dehulled but unmilled rice) length (BRL), width (BRW) and thickness (BRT), but was negatively correlated with the ratio of length to width (RLW). The conditional analysis showed that weight of brown rice (WBR) negatively affected the genetic relationships between $A C$ and the appearance quality traits of rice except between $A C$ and $B R W$, while the genetic relationships between $A C$ and most appearance quality traits were negatively affected by protein content (PC). However, these influences were not apparent due to the impact of WBR or PC on the most covariance components of the different genetic systems between $\mathrm{AC}$ and the appearance quality traits. The conditional analysis showed that it was possible to improve AC while significantly reduce BRL and BRT under maintaining WBR. Furthermore, AC could be improved when BRL was reduced under maintaining PC, but BRW and BRT could be significantly increased.
\end{abstract}

Key words: amylose content, genetic relationships, conditional and unconditional analysis, indica rice quality traits.

Received: August 27, 2007; Accepted: October 15, 2007.

\section{Introduction}

Rice (Oryza sativa L.) is one of the major staple foods, with amylose content (AC) being considered to be one of the most important traits related to the cooking quality of rice. Rice grain shape traits related to the appearance quality of rice are very important in the international market, which usually include length, width, thickness, length to width ratio and length to thickness ratio. Hence, understanding the gene expression and the genetic relationships between $\mathrm{AC}$ and the appearance quality traits (AQT) of rice under different environments is necessary for improving both the cooking quality and the appearance quality of rice.

The relationships between the cooking quality and the appearance quality of rice has been well documented, with some studies having shown a negative correlation between AC and AQT (Sood and Siddiq, 1986; Chauhan et al., 1995; Zhao and Xu, 1998; Feng, 1998), while other studies have reported positive correlations between these pairwise traits in rice (Guo et al., 1985; Wang et al., 1992; Chen et al., 1997; Yang et al., 2001). Hussain et al. (1987) reported that the correlation coefficient between protein content

Send correspondence to Chun-Hai Shi. Department of Agronomy, Zhejiang University, Hangzhou 310020, China. E-mail: chhshi@ zju.edu.cn.
(PC) and grain width was $r=-0.881(\mathrm{p}=0.05)$ while between $\mathrm{PC}$ and the ratio of grain length to grain width $r=-0.344(\mathrm{p}=0.05)$. Shi and Zhu (1994a) pointed out that in indica rice the endosperm and maternal plant genetic effect components were significant between $\mathrm{AC}$ and rice width or the ratio of length to width in indica rice. The relationships between $\mathrm{AC}$ and $\mathrm{AQT}$ of rice might be influenced by the weight of brown rice (WBR) and PC, since AC of rice could be affected as PC or WBR increased (Guo et al., 1985; Hussain et al., 1987; Wang et al., 2005).

Although rice quality traits could be affected by the genes of the triploid endosperm, the rice grain is a new generation that differs from its diploid maternal plant which provides the nutrients for the grain development, and, as such, cytoplasmic effect from cytoplasmic genes and maternal effects from the diploid maternal plant nuclear genes are important components of genetic effects for the performance of rice quality traits. Shi and Zhu (1993, 1994b, 1996) found that rice quality traits were subject to significant seed and maternal genetic effects, while Lin et al. (2005) reported that the AC of japonica rice was not only greatly influenced by the genetic main effects from endosperm, cytoplasm and maternal plant genes but was also affected by genotype environment (GE) interaction effects, because the diversification of gene expression occurred in 
different environments (Bao and Xia, 1999; Shi et al., 1997, 1999). However, genetic effects and relationships between rice quality traits are complex but in the studies discussed above were all directly analyzed using methods based on unconditional analysis, which could not eliminate additional influences from other correlative traits which could affect the relationship between the pairwise traits. For example, the genetic correlations between $\mathrm{AC}$ and AQT of rice might be influenced by WBR or PC, but until now little information has been reported on these factors.

In present experiment, the unconditional analysis and the conditional analysis techniques were used to examine the genetic relationships from different genetic factors (triploid endosperm genes, cytoplasmic genes and diploid maternal plant genes) between $\mathrm{AC}$ and $\mathrm{AQT}$ for indica rice. The influences from WBR or PC of rice to the genetic relationships between $\mathrm{AC}$ and $\mathrm{AQT}$ could be disclosed, which could be helpful in understanding the complex relationships between $\mathrm{AC}$ and $\mathrm{AQT}$ of indica rice.

\section{Materials and Methods}

\section{Field experiment}

We used a factorial mating design involving 12 Oryza sativa (L.) indica rice lines consisting of 7 cytoplasm male sterile (CMS, or A) lines (Zhexie 2, Xieqingzao, Zhenan 3, Zhenshan 97, Gangchao 1, V 20 and Zuo 5) used as females and 5 restorer (R) lines (T 49, Cezao 2-2, 26715, 102 and 1391) used as males. The lines were a random sample of parents for a reference population of indica rice, all seed material coming from paddy field in Hangzhou, China. All CMS female parent lines were crossed to five restorer lines to produce $F_{1}$ seeds in 1997 . The derived $F_{1}$ hybrids, together with their parents, were grown in randomized complete plots with two replications at the experimental farm $\left(120^{\circ} 11^{\prime} 27^{\prime \prime} \mathrm{E}, 30^{\circ} 16^{\prime} 28^{\prime \prime} \mathrm{N}\right.$, altitude $12.5 \mathrm{~m}$, semitropical climate, silt loam soil) of Zhejiang University, Hangzhou, China in 1998 and 1999, respectively. In both years, the seeds were sown on 30 March after germination and the 31-day old seedlings were transplanted with a single plant per round and spaced at $20 \mathrm{~cm}$ within a row. Each plot consisted of 6 rows with 36 plants. The agricultural practices including the prevention of diseases and insects were followed as other standard paddy fields during the course of the experiments. $\mathrm{F}_{1}$ seeds from $\mathrm{A} \times \mathrm{R}$ crosses used in the analysis were produced by pollinating CMS lines with restorers during the same flowering season. Seeds of both parents and the $F_{2}$ offsprings from $F_{1}$ plants were sampled from a batch of 16 mature plants in the middle of each plot. All brown rice was gained from rice grain, which was de-hulled using a sample dehusker (model JLGJ4.5, Taizhou Liangyi Company, China) and milled by sample miller (Model JNMJ3, Taizhou Liangyi Company, China) before grinding to 100-mesh in a model 3010-019 cyclone grinder (Fort Collins, Colorado, USA). The AC and PC of the rice were measured by near-infrared reflectance spectroscopy using a model 5000 monochromator (NIR Systems Inc, Silver Spring, MD, USA) and $3 \mathrm{~g}$ of ground brown rice in a $3.6 \mathrm{~cm}$ ring cup (Wu et al., 2002), all samples being duplicated. The weight of brown rice (WBR), the brown rice length (BRL), the brown rice width (BRW), the brown rice thickness (BRT), the ratio of brown rice length to width (RLW) and the ratio of brown rice length to thickness (RLT) were measured using mature de-husked brown rice, all samples being triplicated.

\section{Statistical methods}

The estimated unconditional phenotypic covariance $\left(C_{P(\mathrm{~T} 1)}\right)$ components between $\mathrm{AC}$ and $\mathrm{AQT}$ of brown rice consisted of the unconditional genetic main covariances $\left(C_{G(\mathrm{~T} 1)}\right)$ and their unconditional GE interaction covariances $\left(C_{G E(\mathrm{~T} 1)}\right)$ and also the unconditional residual covariance $\left(C_{e}\right.$ (T1), which were estimated by the genetic models and statistical methods for quantitative traits of endosperm in cereal crops (Zhu and Weir, 1994). The $C_{G(\mathrm{~T} 1)}$ has the components of the unconditional endosperm additive main covariance $\left(C_{A e}(\mathrm{~T} 1)\right)$, the unconditional endosperm dominance main covariance $\left(C_{D e(\mathrm{~T} 1)}\right)$, the unconditional cytoplasmic main covariance $\left(C_{C}(\mathrm{~T} 1)\right)$, the unconditional maternal additive main covariance $\left(C_{A m(\mathrm{~T} 1)}\right)$, the unconditional maternal dominance main covariance $\left(C_{D m \text { (T1) }}\right)$, the unconditional covariance between endosperm and maternal additive effects $\left(C_{A e \cdot A m \text { (T1) }}\right)$, the unconditional covariance between endosperm and maternal dominance effects $\left(C_{D e \cdot D m(\mathrm{~T} 1)}\right) \cdot C_{G E(\mathrm{~T} 1)}$ has the components of $C_{A e E(\mathrm{~T} 1)}, C_{D e E(\mathrm{~T} 1)}, C_{C E(\mathrm{~T} 1)}, C_{A m E(\mathrm{~T} 1)}$, $C_{D m E(\mathrm{~T} 1)}, C_{A e E \cdot A m E(\mathrm{~T} 1)}$ and $C_{D e E \cdot D m E(\mathrm{~T} 1)}$.

The mixed model approach of conditional genetic analysis for quantitative traits ( $\mathrm{Zhu}, 1995)$ was used to obtain the conditional phenotypic covariances $\left(C_{P}\right.$ (T1| T2) $)$ between $\mathrm{AC} \mid \mathrm{WBR}$ or $\mathrm{AC} \mid \mathrm{PC}$ and the $\mathrm{AQT}$ of brown rice. Here, T1|T2 denotes trait 1 conditioned on trait 2, which means the conditional phenotypic value of traits 1 , when excluded the impacts of trait 2 on trait $1, \mathrm{AC} \mid \mathrm{WBR}$ or $\mathrm{AC} \mid \mathrm{PC}$ means, hence, $\mathrm{AC}$ conditioned on WBR or PC, respectively. The conditional phenotypic covariance $\left(C_{P(\mathrm{~T} \mid \mathrm{T} 2)}\right)$ components including the conditional endosperm additive main covariance of trait 1 conditioned on trait 2 $\left(C_{A e}\right.$ (T1|T2) $)$, the conditional endosperm dominance main covariance of trait 1 conditioned on trait $2\left(C_{D e \text { (T1|T2) }) \text {, the }}\right.$ conditional cytoplasm main covariance of trait 1 conditioned on trait $2\left(C_{C(\mathrm{~T} 1 \mid \mathrm{T} 2)}\right)$, the conditional maternal additive main covariance of trait 1 conditioned on trait $2\left(C_{A m}\right.$ $(\mathrm{T} 1 \mid \mathrm{T} 2)$ ), the conditional maternal dominance main covariance of trait 1 conditioned on trait $2\left(C_{D m \text { (T1|T2) })}\right)$, the conditional covariance between endosperm and maternal additive effects of trait 1 conditioned on trait $2\left(C_{A e \cdot A m}\right.$ $(\mathrm{T} 1 \mid \mathrm{T} 2)$ ), the conditional covariance between endosperm and maternal dominance effects of trait 1 conditioned on trait 2 $\left(C_{D e \cdot D m(\mathrm{~T} 1 \mid \mathrm{T} 2)}\right)$, and their conditional GE interaction covariances $C_{A e E \text { (T1|T2) }}, C_{D e E(\mathrm{~T} 1 \mid \mathrm{T} 2)}, C_{C E \text { (T1|T2) }}, C_{A m E \text { (T1|T2) }}, C_{D m E}$ 
(T1|T2), $C_{A e E \cdot A m E ~(T 1 \mid \mathrm{T} 2)}, C_{D e E \cdot D m E}(\mathrm{~T} 1 \mid \mathrm{T} 2)$. And the conditional phenotypic covariance $\left(C_{P}\right.$ (T1|T2) $)$ also including the conditional residual covariance of trait 1 conditioned on trait 2 $\left(C_{e}\right.$ (TI|T2) $)$. The Jackknife re-sampling method was used with sampling generation means of entries to derive the standard errors of estimated components of unconditional or conditional covariances.

\section{Results and Discussion}

\section{Covariance analysis between $\mathrm{AC}$ and WBR or PC}

All the phenotypic covariances $\left(C_{P}\right)$ and genotypic covariances $\left(C_{G}\right)$ between $\mathrm{AC}$ and WBR or $\mathrm{AC}$ and $\mathrm{PC}$ were significant (Figure 1), indicating that there was true genetic association between $\mathrm{AC}$ and WBR or PC because the magnitude of genetic covariance for all the pairwise traits was similar to or larger than the phenotypic covariance between AC and WBR or AC and PC. None of the estimated residual covariances $\left(C_{e}\right)$ were significant, so the influences from the sampling errors and microenvironmental factors were not important for the genetic relationships between AC and WBR or AC and PC. Hence, WBR or $\mathrm{PC}$ had a close genetic relationship with the $\mathrm{AC}$ of indica rice, and the conditional analysis method could be used for analyzing the conditional genetic relationship between $\mathrm{AC}$ and other traits of indica rice.

\section{Phenotypic and genotypic covariances between AC and AQT}

For the unconditional analysis, $C_{P(\mathrm{AC})}$ and $C_{G(\mathrm{AC})}$ values for most of AQT were significantly positive or negative, except for the covariances between AC and BRL or RLT (Table 1). For example, $C_{G(\mathrm{AC})}$ value was 18.09 $(\mathrm{p}=0.05)$ for BRW, $8.58(\mathrm{p}=0.05)$ for BRT and -16.13 $(\mathrm{p}=0.05)$ for RLW. Although the $C_{P(\mathrm{AC})}$ for BRL was not significant $\left(C_{P(\mathrm{AC})}=6.32\right), C_{G(\mathrm{AC})}$ was significantly positive for BRL $\left(C_{G(\mathrm{AC})}=8.53, \mathrm{p}=0.05\right)$ and the genotypic relationship between this pair of traits was clear. It was obvious that AC was positively correlated to most of AQT of rice, except for the RLW and RLT traits. The estimated $C_{e(\mathrm{AC})}$ values for BRL and BRW both significant, indicating that the relationship between $\mathrm{AC}$ and both these traits was also influenced by the sampling errors or factors related to

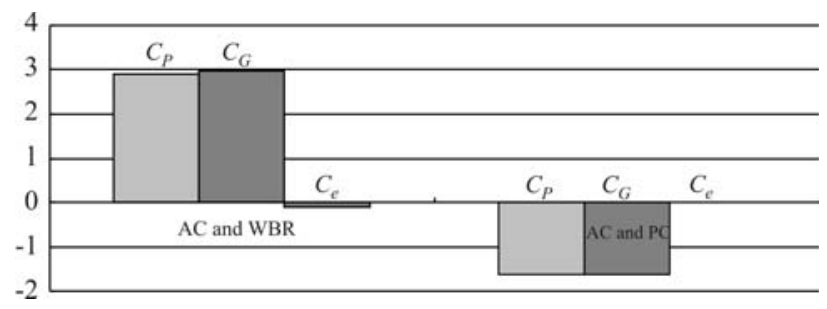

Figure 1 - The unconditional analysis covariance components between amylose content $(\mathrm{AC})$ and weight of brown rice (WBR) or $\mathrm{AC}$ and protein content $(\mathrm{PC})$. Figure shows phenotypic covariance $\left(C_{P}\right)$, genotypic covariance $\left(C_{G}\right)$ and residual covariance $\left(C_{e}\right)$. the microenvironment. The small $C_{e(\mathrm{AC})}$ values for these pairwise traits indicated that the correlations between $\mathrm{AC}$ and other AQT were mainly controlled by the genetic effects. This suggests that improving AC could simultaneously increase BRL, BRW and BRT.

Since the relationships between $\mathrm{AC}$ and AQT could be significantly influenced by WBR or PC (Figure 1), the conditional analysis method was used to further detect the genetic correlations between the pairwise traits between $\mathrm{AC}$ and AQT. When AC was conditional on WBR (AC|WBR), the conditional phenotypic covariance $\left(C_{P(\mathrm{AC} \mid \mathrm{WBR})}\right)$ and the conditional genotypic covariance $\left(C_{G(\mathrm{AC} \mid \mathrm{WBR})}\right)$ between $\mathrm{AC} \mid \mathrm{WBR}$ and $\mathrm{BRL}$ or $\mathrm{AC} \mid \mathrm{WBR}$ and BRT changed to significantly negative (Table 1 ). $C_{P(\mathrm{AC} \mid \mathrm{WBR})}$ and $C_{G(\mathrm{AC} \mid \mathrm{WBR})}$ between $\mathrm{AC} \mid \mathrm{WBR}$ and $\mathrm{BRW}$ ceased to be significantly positive as compared to the significantly positive $C_{P(\mathrm{AC})}$ and $C_{G(\mathrm{AC})}$ values produced by unconditional analysis. $C_{P(\mathrm{AC} \mid \mathrm{WBR})}$ and $\left(C_{G(\mathrm{AC} \mid \mathrm{WBR})}\right)$ between $\mathrm{AC} \mid \mathrm{WBR}$ and RLW or RLT were significantly negative and the values were larger than those produced by unconditional analysis. It therefore appears that the influence of WBR on the relationships between $\mathrm{AC}$ and $\mathrm{AQT}$ was complex in that WBR could positively affect the relationship between $\mathrm{AC}$ and

Table 1 - Estimated phenotypic and genotypic covariance components from the unconditional and conditional analysis between amylose content (AC) and the appearance quality traits (AQT), including brown rice length (BRL), brown rice width (BRW), brown rice width (BRT), ratio of brown rice length to width (RLW) and ratio of brown rice length to thickness (RLT).

\begin{tabular}{lccccc}
\hline \multirow{2}{*}{$\begin{array}{l}\text { Covariance } \\
\text { parameters }\end{array}$} & \multicolumn{5}{c}{ Appearance quality traits } \\
\cline { 2 - 6 } & $\mathrm{BRL}$ & $\mathrm{BRW}$ & $\mathrm{BRT}$ & $\mathrm{RLW}$ & $\mathrm{RLT}$ \\
\hline$C_{P(\mathrm{AC})}$ & 6.32 & $17.53^{*}$ & $8.41^{*}$ & $-16.38^{*}$ & -12.69 \\
$C_{P(\mathrm{AC} \mid \mathrm{WBR})}$ & $-65.19^{* *}$ & 5.68 & $-6.43^{*}$ & $-33.20^{* *}$ & $-22.65^{* *}$ \\
$C_{P(\mathrm{AC} \mid \mathrm{PC})}$ & $-27.97^{*}$ & $28.25^{* *}$ & $8.34^{* *}$ & $-42.53^{* *}$ & $-31.16^{* *}$ \\
$C_{G(\mathrm{AC})}$ & $8.53^{*}$ & $18.09^{*}$ & $8.58^{*}$ & $-16.13^{*}$ & -11.78 \\
$C_{G(\mathrm{AC} \mid \mathrm{WBR})}$ & $-63.70^{* *}$ & 5.94 & $-6.44^{*}$ & $-32.90^{* *}$ & $-21.82^{* *}$ \\
$C_{G(\mathrm{AC} \mid \mathrm{PC})}$ & $-25.85^{*}$ & $28.94^{* *}$ & $8.60^{* *}$ & $-42.46^{* *}$ & $-30.48^{* *}$ \\
$C_{e(\mathrm{AC})}$ & $-2.22^{* *}$ & $-0.56^{* *}$ & -0.17 & -0.26 & -0.91 \\
$C_{e(\mathrm{AC} \mid \mathrm{WBR})}$ & -1.49 & -0.25 & 0.01 & -0.30 & -0.84 \\
$C_{e(\mathrm{AC} \mid \mathrm{PC})}$ & -2.12 & -0.69 & -0.27 & -0.07 & -0.68 \\
\hline
\end{tabular}

${ }^{\dagger} \mathrm{WBR}$, weight of brown rice; $\mathrm{PC}$, protein content; $C_{P(\mathrm{AC})}$, unconditional covariance between $\mathrm{AC}$ and the appearance quality traits; $C_{P(\mathrm{AC} \mid \mathrm{WBR})}$, conditional phenotypic covariance between $\mathrm{AC} \mid \mathrm{WBR}$ and the appearance quality traits; $C_{P(\mathrm{AC} \mid \mathrm{PC})}$, conditional phenotypic covariance between $\mathrm{AC} \mid \mathrm{PC}$ and the appearance quality traits; $C_{G(\mathrm{AC})}$, unconditional genotypic covariance between $\mathrm{AC}$ and the appearance quality traits; $C_{G(\mathrm{AC} \mid \mathrm{WBR})}$, conditional genotypic covariance between $\mathrm{AC} \mid \mathrm{WBR}$ and the appearance quality traits; $C_{G(\mathrm{AC} \mid \mathrm{PC})}$, conditional genotypic covariance between $\mathrm{AC} \mid \mathrm{PC}$ and the appearance quality traits; $C_{e(\mathrm{AC})}$, unconditional residual covariance be-

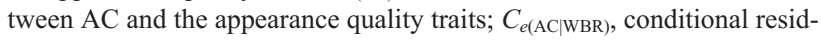
ual covariance between $\mathrm{AC} \mid \mathrm{WBR}$ and the appearance quality traits; $C_{e(\mathrm{AC} \mid \mathrm{PC})}$, conditional residual covariance between $\mathrm{AC} \mid \mathrm{PC}$ and the appearance quality traits.

*Significant at the $\mathrm{p}=0.05$ by the t-test.

** Significant at the $\mathrm{p}=0.01$ by the $\mathrm{t}$-test. 
BRW but negatively affect the relationship between $\mathrm{AC}$ and the other appearance quality traits. While $\mathrm{AC}$ was conditional on $\mathrm{PC}(\mathrm{AC} \mid \mathrm{PC})$, all $C_{P(\mathrm{AC} \mid \mathrm{PC})}$ and $C_{G(\mathrm{AC} \mid \mathrm{PC})}$ between $\mathrm{AC} \mid \mathrm{PC}$ and $\mathrm{AQT}$ were significantly positive or negative (Table 1). Among them, $C_{P(\mathrm{AC} \mid \mathrm{PC})}$ and $\left(C_{G(\mathrm{AC} \mid \mathrm{PC}}\right)$ between $\mathrm{AC} \mid \mathrm{PC}$ and $\mathrm{BRL}$ became significantly negative, while those between AC|PC and BRW, BRT, RLW, RLT were all significantly positive or negative by conditional analysis. However, the conditional analysis values were generally notably larger than those produced by unconditional analysis, the exception being the pairwise traits between AC|PC and BRT, which were similar to the unconditional analysis values. It was found that PC could negatively affect most of the genetic relationships between $\mathrm{AC}$ and the appearance quality traits, especially for the relationship between $\mathrm{AC}$ and BRL. Since none of the small $C_{e(\mathrm{AC} \mid \mathrm{WBR})}$ or $C_{e(\mathrm{AC} \mid \mathrm{PC})}$ values from the conditional analysis were significant, the conditional genetic correlations between AC and AQT were not influenced by sampling errors or microenvironmental factors when $\mathrm{AC}$ was conditioned on WBR or PC.

\section{Analysis of AC and AQT covariance components from different genetic systems}

Since rice quality traits were simultaneously controlled by the genes from the triploid endosperm, cytoplasm and diploid maternal plant, the genetic main effects as well as their GE interaction effects were important for the performance of rice quality. The covariance components from the different genetic systems between AC and AQT could be evaluated by the unconditional analysis method and conditional analysis method (Table 2).

The unconditional analysis results in Table 2 shows that $C_{A e}$ (AC) between $\mathrm{AC}$ and BRL or AC and RLW was significantly negative, while $C_{C}$ (AC) between $\mathrm{AC}$ and $\mathrm{BRL}$ or $\mathrm{AC}$ and $\mathrm{BRW}$ and $C_{A e \cdot A m}$ (AC) between $\mathrm{AC}$ and $\mathrm{BRL}$ were significantly positive. For the GE interaction covariance, only $C_{D e E ~(A C)}$ and $C_{D e E \cdot D m E ~(A C)}$ between AC and RLT were significantly negative. Since other covariance parameters from different genetic systems between AC and AQT were not significant, the genetic relationships from endosperm, cytoplasmic and maternal plant effects were not visible for most of the pairwise traits between AC and AQT.

For the conditional analysis, when AC was conditional on WBR (AC|WBR) almost all the covariance components from the different genetic systems between $\mathrm{AC} \mid \mathrm{WBR}$ and AQT were not significant, except for the significant positive $C_{A e E(\mathrm{AC} \mid \mathrm{WBR})}$ and significant negative $C_{\text {DeE(AC|WBR) }}$ between $\mathrm{AC} \mid \mathrm{WBR}$ and RLT (Table 2.). For $\mathrm{AC}$ conditional on $\mathrm{PC}(\mathrm{AC} \mid \mathrm{PC})$, the GE interaction covariances including $C_{D m E(\mathrm{AC} \mid \mathrm{PC})}, C_{D e E-D m E(\mathrm{AC} \mid \mathrm{PC})}$ between $\mathrm{AC} \mid \mathrm{PC}$ and BRT or $C_{D e E-D m E(\mathrm{AC} \mid \mathrm{PC})}$ between $\mathrm{AC} \mid \mathrm{PC}$ and RLT were significantly correlated. The influence of WBR or PC on the covariances from different genetic system was not large, because only small endosperm, cytoplasmic or maternal covariance parameters were detected significant between $\mathrm{AC}$ and AQT by conditional analysis method.

Most rice quality traits are complex quantitative traits, which are not only controlled by genetic main effects, GE interaction effects and environmental conditions but can also be influenced by other traits. Hence, analyzing the genetic correlations for pairwise quality traits of rice should consider these factors. In general, the quantitative traits can affect each other in the developmental period of rice, which also can interfere with the genetic relationships between the quality traits. To understand the true genetic correlations between rice quality traits, it is better to exclude the extra factors which can affect the relationship between pairwise traits. Although some genetic models and statistical analysis methods have been proposed for analyzing the genetic correlations between complex quantitative traits they do not effectively exclude the influences of the other trait(s) on the relationship between two quantitative traits (Mo, 1985; Zhu, 1992; Zhu and Weir, 1994). Hence, the choice of genetic models and statistical analysis methods are very important when researching quantitative traits. Zhu (1995) proposed a new statistical method for analyzing conditional genetic effects and conditional genetic variance components based on the genetic models for quantitative traits with contribution measure for each component to the complex traits. By using this conditional analysis method, the causal influences from the extra trait which affected the genetic relationships between AC and AQT of rice can be excluded and the actual genetic correlation between the pairwise traits can be found. This conditional analysis method can also estimate the genetic main effects as well as their GE interaction effects from the triploid endosperm, cytoplasm and diploid maternal plants.

Our results presented in present experiment indicate that under the influenced of WBR or PC, AC was positively correlated to BRL, BRW or BRT, but was negatively correlated to RLW or RLT. The results of conditional analysis revealed that WBR could positively affect the relationship between AC and BRW, but negatively affect the relationships for other pairwise traits, while PC could negatively affect the relationships between $\mathrm{AC}$ and most of the appearance quality traits. Among the genetic correlations from different genetic systems including endosperm, cytoplasmic and maternal effects for the pairwise quantitative traits, only some significant covariance components were detected between AC and AQT of rice analyzed by both the unconditional or conditional methods, with WBR or PC having little impact on the covariance components of different genetic systems between the pairwise traits of indica rice.

Comparing the results from the unconditional and conditional analysis, it appears that, for the same brown rice weight, it is possible to improve the amylose content and simultaneously noticeably reduce the brown rice length and brown rice thickness. Furthermore, if breeders improve 


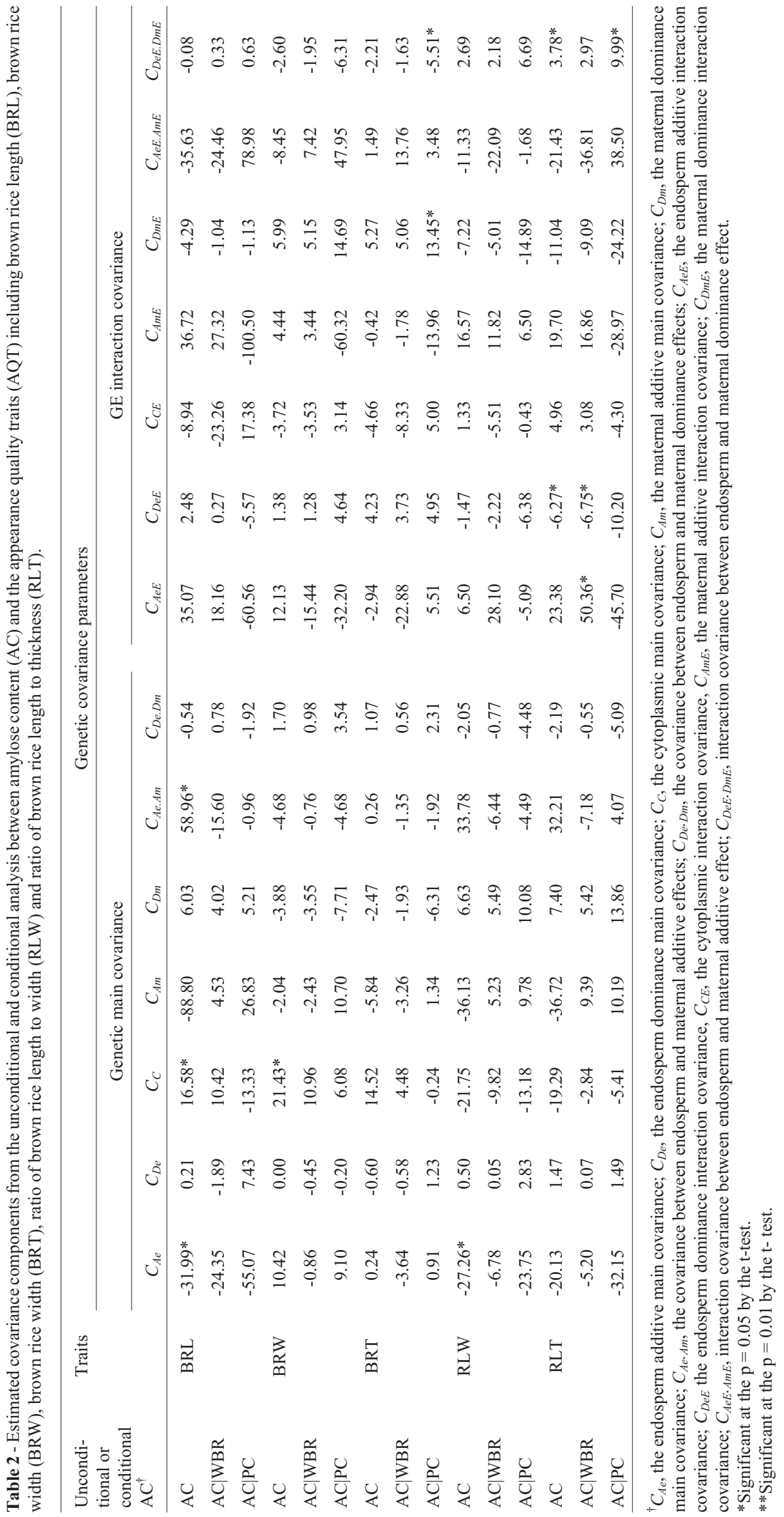


the rice amylose content while maintaining the same protein content, the brown rice length could be reduced while simultaneously increasing the brown rice width and the brown rice thickness.

The mixed model approaches of conditional genetics analysis for quantitative traits described in present paper provides a new analysis method and strategy for research on the relationship between quantitative traits and might be useful not only for rice but for other cereal crops.

\section{Acknowledgments}

The project is financially supported by the National Basic Research Program of China (973 Program, n. 2007CB109000), the National Natural Science Foundation of China (n. 30571198) and the Science and Technology Office of Zhejiang Province (n. 011102471).

\section{References}

Bao JS and Xia YW (1999) Genetic control of viscosity characteristics in indica rice (Oryza sativa L.). Theor Appl Genet 98:1120-1124.

Chauhan JS, Chauhan VS and Lodh SB (1995) Comparative analysis of variability and correlations between quality components in traditional rainfed upland and lowland rice. Indian J Genet Plant Breed 55:6-12.

Chen N, Luo YK, Zhu ZW, Zhang BP, Zheng YC and Xie LH (1997) Correlation between eating quality and physico-chemical properties of high grain quality rice. Chinese J Rice Sci 11:70-76.

Feng YX (1998) Analyzed of the innovation and utilize for the varieties resource of high quality indica rice. Hunan Agric Sci 5 4-8.

Guo YQ, Liu Q, Zhang DM and Zhang SJ (1985) Genetics analysis between the cooking quality and the eating quality, traits of grains for indica rice. Agric Res China 34:243-257.

Hussain AA, Maurya DM and Vaish CP (1987) Study on quality status of indigenous upland rice (Oryza sativa L.). Indian J Genet 47:145-152.

Lin JR, Shi CH, Wu MG and Wu JG (2005) Analysis of genetic effects for cooking quality traits of japonica rice across environments. Plant Sci 168:1501-1506.

Mo HD (1985) Genetic analysis of covariance. J Jiangsu Agric Univ 6:51-56.
Shi CH, Xue JM, Yu YG, Yang XE and Zhu J (1996) Analysis of genetic effects for nutrient quality traits in indica rice. Theor Appl Genet 92:1099-1102.

Shi CH and Zhu J (1993) Analysis of seed and maternal genetic effects for exterior quality characters in hybrids of indica rice. Acta Agri Univ Pekinensis 19:69-74.

Shi CH and Zhu J (1994a) Correlations analysis of rice exterior quality traits and other traits in indica rice. J Zhejiang Agri Univ 20:606-610.

Shi CH and Zhu J (1994b) Analysis of seed and maternal genetic effects for characters of cooking quality in indica rice. Chin $\mathrm{J}$ Rice Sci 8:29-134.

Shi CH, Zhu J, Yang XE, Yu YG and Wu JG (1999) genetic analysis for the protein content in indica rice. Euphytica 107:135-140.

Shi CH, Zhu J, Zang RC and Chen GL (1997) Genetic and heterosis analysis for cooking quality traits of indica rice in different environments. Theor Appl Genet 95:294-300.

Sood BC and Siddiq EA (1986) Possible physico-chemical attributes of kernel influencing kernel elongation in rice. Genetics 46:456-460

Wang DY, Zhang XF, Zhu ZW, Chen N, Min J, Yao Q, Yan JL and Liao XY (2005) Correlation analysis of rice grain quality characteristics. Acta Agronomic Sin 31:1086-1091.

Wang JL, Xiong ZM and Zhu XD (1992) Study on the correlation and the heterosis among the rice quality characters for the F1 of indica-japonica hybrid rice. Bull Rice Breed 12:20-26.

Wu JG, Shi CH, Zhang XM and Fan LJ (2002) Estimating the amino acid composition in the milled rice powder by nearinfrared reflectance spectroscopy. Field Crops Res 75:1-7.

Yang LS, Bai YS, Zhang PJ, Xu CW, Hu XM, Wang WM, She DH and Chen GZ (2001) Studies on the correlation between grain shape and grain quality in rice. Hybrid Rice 16:48-54.

Zhao ZH and Xu YH (1998) The evaluated and analyzed of the high quality varieties and the standard systematic for Hunan province's rice. Hunan Agric Sci 2:6-9.

Zhu J (1992) Mixed model approaches for estimating genetic variances and covariances. J Biomath 7:1-11.

Zhu J (1995) Analysis of conditional effects and variance components in developmental genetics.Genetics 141:1633-1639.

Zhu J and Weir BS (1994) Analysis of cytoplasmic and maternal effects: II. Genetic models for triplod endosperms. Theor Appl Genet 89:160-166.

Senior Editor: Ernesto Paterniani

License information: This is an open-access article distributed under the terms of the Creative Commons Attribution License, which permits unrestricted use, distribution, and reproduction in any medium, provided the original work is properly cited. 